\title{
Energy production control of an experimental kite system in presence of wind gusts.
}

\author{
Rogelio Lozano Jr, Jonathan Dumon, Ahmad Hably and Mazen Alamir
}

\begin{abstract}
The growing need of energy, global warming and recent nuclear power plant accidents have shown that renewable energies need to be developed for tomorrow's world. Wind energy is generally harvested using wind turbines. Unfortunately, these systems have some drawbacks such as their cost, and the amount of steel and concrete used for construction. As their size grows, their complexity increases exponentially. This paper studies an alternative solution for the production of wind energy, using a kite's traction force. The aim of this paper is to control the amount of energy produced by the kite, and to be able to fly it safely in the presence of strong wind gusts. Our theoretical work has been implemented in a scale model flying autonomously in a wind tunnel. The proposed control strategy has led to control the system output power with an accuracy greater than $95 \%$, with unknown wind speeds varying from $\mathbf{7 . 5}$ to $9 \mathrm{~m} / \mathrm{s}$.
\end{abstract}

\section{INTRODUCTION}

Energy kites systems have been studied for the last 10 years. There have been mainly theoretical works about the automatic control of the trajectory [12 - 19]. Few papers have validated their theoretical results with a flying prototype. The main reasons are that it is complex, costly and usually, prototypes have to be rebuilt once they crash. This system produces energy during cycles in which the kite moves away from, and returns to it's initial point, following a straight trajectory. As the kite moves away lifted by the wind, the wing's angle of attack is kept at an angle which produces an important lift force. The tether displacement makes a dynamo on the ground turn, which generates power. During the period when the kite is descending, the angle of attack is reduced so that the lift force is significantly smaller, and the dynamo is used as a motor for reeling back the kite to its initial altitude. As a consequence, few energy is used reeling the kite back and there will be a positive amount of energy generated at the end of each cycle. Most studies have focused on different kinds of trajectories, called crosswind flight, that maximizes the energy produced per kite. Its main drawback is that each kite requires a lot of room to fly, as it follows a 'figure- 8 ' trajectory. The system studied in this paper has a kite that performs ascending and descending trajectories along the same straight trajectory. This system has two advantages over the 'crosswind kites'. While it does generate a lower amount of energy per kite, since the flight space of each kite is a straight trajectory, one can put several systems in a very small land area. As a consequence, this system increases the amount of energy produced per used

Automatic control department, GIPSA-lab ENSE3, Domaine universitaire, 11 rue des Mathmatiques, BP 46, 38402 Saint-Martin d'Heres, France Rogelio.Lozanodgipsa-lab.grenoble-inp.fr ground surface. It is better adapted to sites where the land used is a strong constraint that needs to be optimized. The second advantage is that it is safer. In order to have increased performances, crosswind kites need to have a larger aspect ratio. As a consequence, they need to have a strong structure that will be stiff and heavy. Our system does not need to have a high aspect ratio and can be built with soft materials.

This paper has three main objectives: - Stability against perturbations is a crucial point. Wind gusts can be very brief and of enough intensity that they might cause loss of control of the kite before the actuators have time to react. For this reason, the flight plan needs to be studied so that it is wind gust-proof.

- To control the output power so that the generated energy can be plugged directly into the grid without the need of batteries or supercapacitors. This proposed control strategy also extends the lifetime of the system.

- To propose a methodology for those who would like to build their own prototypes. This methodology has been inspired by aviation pioneers and UAV research.

Moreover, the previous points can be used for other kinds of kites systems.

\section{The Guiding Principles For This Study}

The following guidelines sum up techniques and methodologies inspired by the history of aviation [1 - 9], flight mechanics [10], and by the team's experience in the domain of UAV control. They tend to reduce crashes, simplify experimental validation, and give better results faster.

\section{A. Perform tests in a wind tunnel experimental setup}

Being independent of weather conditions makes our research go much faster. We can control the wind speed, we can fly anytime and we do not have to transport equipment to perform flight tests. We can also perform static flights, study aerodynamic coefficients, simulate perturbations, block it with the hand, be next to it as it is flying, avoid crashes, etc.

\section{B. Minimal correlation of controls}

Generally, flight parameters are correlated. For example, a kitesurf's pitch angle depends on the flight angle, its control accuracy on the relative speed of the kite, of the value of the angle of attack, the length of the tether, etc. This kind of characteristics make the control of kite systems much more complex. This study tries to avoid this kind of correlations. 


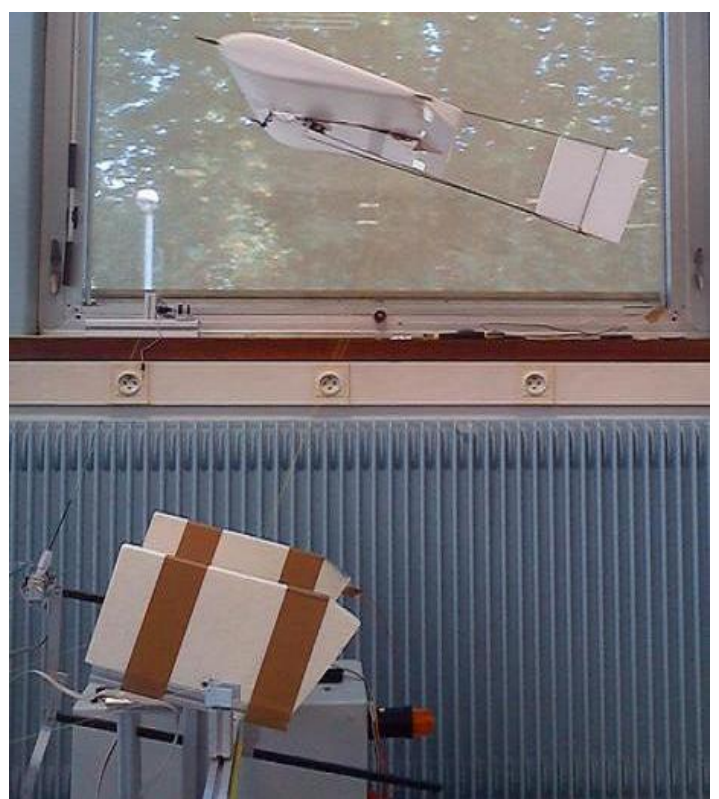

Fig. 1. Flight test of the wind power system in the GIPSA Wind Tunnel (wind speed: $8 \mathrm{~m} / \mathrm{s}$ ).

\section{Study a simple prototype}

Our aim is to work with an increasing level of complexity. As it is our first experience in the kite energy domain, we have chosen to study a relatively simple kite design. Note that all of the principles explained in this paper can be used as a basis for much more complex systems.

\section{The crash-proof concept}

Crashes seem to be an unavoidable part of experimentation. The faster we recover from a crash, the faster we learn to fly without crashing. We have chosen to design a very simple and robust prototype. Its robustness lies in the use of an inner structure of carbon fiber and foam sheets to give the external surface its aerodynamic shape. Its simplicity is such that in case of important damage, the kite can be fully reconstructed or modified within few hours. To date, this prototype has experienced about 30 crashes, but none of them requiring more than half an hour to be repaired.

\section{Description of the Kite Prototype}

A wind tunnel and several kites have been constructed at Gipsa-lab, Grenoble. The following study focuses on our fourth prototype, shown in Figure 1. A symmetric NACA 0018 [[21]] airfoil has been chosen for two main reasons: aerodynamic stall propagation is very progressive and the airfoil has enough room for the embedded electronics. Moreover, its pitch moment at a quarter of the cord is independent of the Angle Of Attack $(\mathrm{AOA}=\alpha)$. Definitions and fundamental aerodynamic knoledge can be read in [10]. The wing shown in Figure 2 has a constant cord, a symmetrical airfoil, two embedded actuators that control the tail rudder and the spoilers, which increase the amount of profile drag. The AOA is controlled using a servo on the ground that pulls a rope attached to the leading edge of the wing and two other ropes that are attached to the trailing edge. This rope system makes the AOA independent of the flight angle $\theta$ (see Figure 3). Definitions

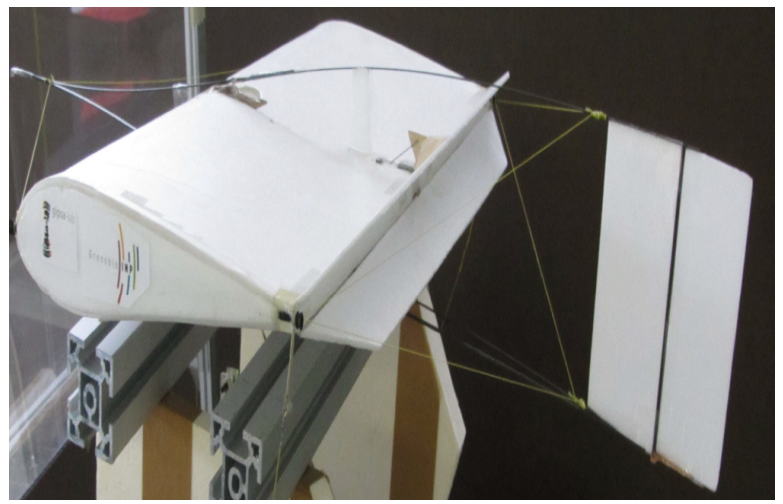

Fig. 2. The wing with the spoilers in a medium drag position. Note the rudder used for the yaw angle stabilization.

As weight is a major problem, we have chosen to mechanically lock the roll angle, the lateral position and the yaw angle. The roll axis is locked by the lift force and a rope system connected to the sides of the wing. The lateral position is also stabilized by the lift force. The yaw axis is stabilized by a PID controller that acts on the tail rudder. Although not detailed in this study, the controller is effective enough to consider the flight as two-dimensional. The trajectory of the kite and the produced power need to be controlled. This trajectory is composed of the flight angle $\theta$ and the rope's length $r$ as depicted in Figure 3.

It has been noticed that at high angles of attack, some very strong perturbations appear on the $\theta$ axis. If they are not properly controlled, these oscillations can lead our kite to crash. The same kind of oscillations can appear on the rope's length $r$ control. The angle of attack $\alpha$ needs to be controlled as well, it has to adapt to changes of relative wind speed and orientation. The angle of attack will be defined here as the sum of the pitch angle $\alpha_{u}$ measured with respect to the horizon, the relative wind orientation $\alpha_{w}$ and its perturbation $\delta_{\alpha w}$ due to wind gusts.

$$
\alpha=\alpha_{u}+\alpha_{w}+\delta_{\alpha w}
$$

\section{WIND CHARACTERISTICS}

Wind gusts can make the orientation and the strength of wind change, and they can be very brief and intense. They are one of the biggest causes of crash in aeronautics, kitesurfing and paragliding. If the wind gust causes the angle of attack to decrease, the lift change can provoke a phenomenon similar to the one known as 'frontal collapse' in paragliding. On the contrary, if the wind gust increases the AOA, it can provoke a stall.

Wind gusts also make the overall wind speed value change. Combined, both phenomena can lead to great variations of lift, which would, in turn cause variations in the output 


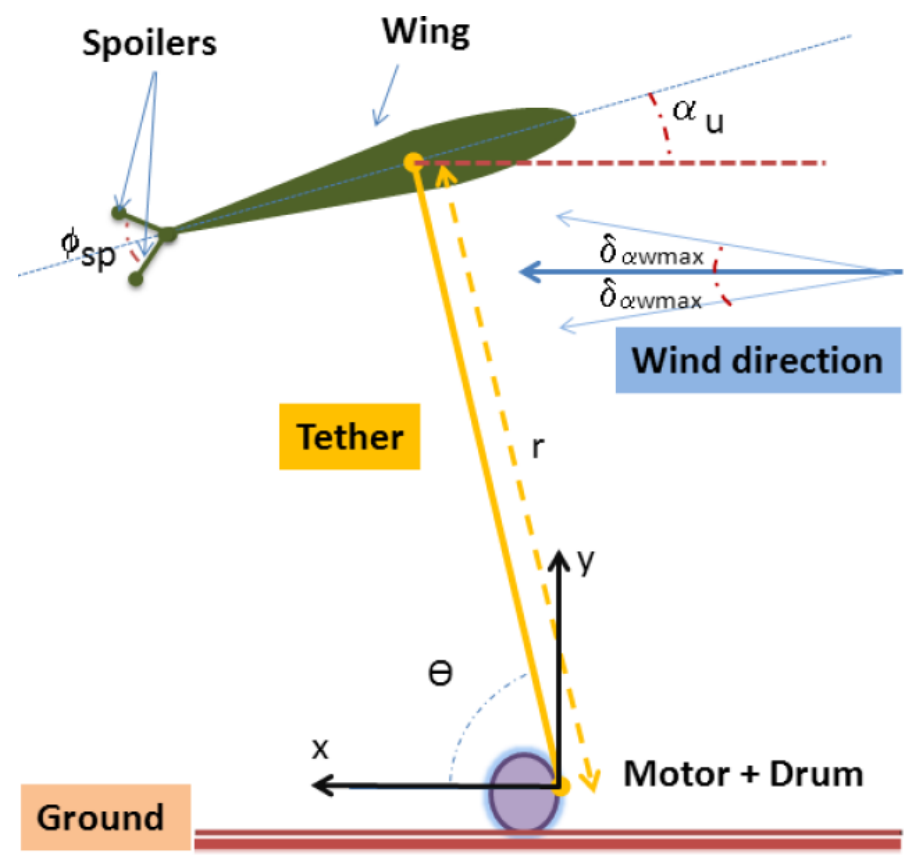

Fig. 3. The whole wind power system (wing+tether+dynamo/motor) in airflow

power. The wind speed $V$ will here be modeled as the sum of a controlled main stream speed $\bar{V}$ and uncontrolled variations of intensity $\delta_{V}$. This wind blows horizontally but wind gusts can change its angle of orientation $\delta_{\alpha w}$.

The wind tunnel's mean wind speed $\bar{V}$ obeys a first order filter that is controlled by the control input $U_{V}$, namely:

$$
\dot{\bar{V}}=\beta_{V}\left(U_{V}-\bar{V}\right)
$$

Nine brushless motors generate a wind speed that goes up to $9 \mathrm{~m} / \mathrm{s}$ with a response time of approximately $0.5 \mathrm{~s}$. This control will be used to simulate wind gusts along the $\mathrm{x}$ axis. During the tests, values of $\bar{V}$ will be of 7,8 and $9 \mathrm{~m} / \mathrm{s}$.

The propellers' turbulence provokes some perturbations that cannot be controlled. Normally, their characteristics depend on the ground's surface and shape, temperature differences, the presence of other flying machines, etc. In this study, wind perturbations are approximated by simple models where one only knows bounds on their amplitudes.

$$
\left\|\delta_{V}\right\|<\delta_{V \max } ;\left\|\delta_{\alpha w}\right\|<\delta_{\alpha w \max }
$$

The GIPSA Wind Tunnel has values of $\left\|\delta_{V}\right\|$ that are smaller than $\delta_{V \max }=0.5 \mathrm{~m} / \mathrm{s}$ and $\left\|\delta_{\alpha w}\right\|$ is bounded by $\delta_{\alpha w \max }=3$ degrees. In this specific case, lateral wind gusts have a very low impact on the system so they will not be taken into account.

\section{Causes of Crashes and Safe Flight Conditions}

The control algorithms must be designed so that the kite respects the four following safe flight conditions.

\section{A. Condition 1: Dynamic stall}

The angle of attack $\alpha$ must never be greater then the stall angle $\alpha_{\text {stall }}$. This dynamic stall condition must be taken into account for the worst possible value of $\delta_{w \alpha}$, i.e. when it is at its maximal value.

$$
\alpha=\alpha_{u}+\alpha_{w}+\delta_{w \alpha}<\alpha_{\text {stall }}
$$

\section{B. Condition 2: Frontal collapse and loss of tension}

Frontal collapse can happen to paragliders when the lift coefficient suddenly becomes too small or negative. If it happens, a classic paraglider's airfoil loses its shape and the lift changes its orientation, leading to a total loss of control. It is the first cause of crashes in paragliding [12]. The second kind of problem happens to kites that need a minimal rope tension in order to have effective control. It is generally the case when the actuators are not embedded. These two phenomena should lead to two different conditions, one on the minimal tension, and one on the minimal angle of attack. However, in this study, the minimal needed rope tension is very small and we will approximate both conditions by the following one. The kite must never fly with an angle of attack lower than $\alpha_{\min }=2$ degrees.

$$
\alpha=\alpha_{u}+\alpha_{w}-\delta_{w \alpha}>\alpha_{m i n}=2 \text { degrees }
$$

\section{Condition 3: Overload}

Our system's lift $\vec{L}$ and drag $\vec{D}$ forces must respect the structure's maximum load $F_{\max }$. Beyond the maximum value, the kite, the rope or the dynamo will be destroyed. This inequality must be respected in the worst case, i.e. when the wind gusts $\delta_{V}$ and the $\delta_{\alpha}$ are at their max value. This study's kite maximum load is $10 \mathrm{~N}$.

$$
\|\vec{L}+\vec{D}\|<F_{\max }
$$

\section{Condition 4: Minimal lift}

The vertical component of aerodynamic forces must be greater than the weight $w=M g$ in the worst case, i.e. when the wind gusts decrease the $V$ speed and the relative wind angle to their minimum value:

$$
\|(\vec{L}+\vec{D}) \cdot \vec{y}\|>M g
$$

\section{Aerodynamic Model}

This section presents the aerodynamic model of a kite wind power generator system as depicted in Figure (3). Aerodynamical formulas are taken from [10]. From Newton's second law we obtain the following nonlinear dynamical system:

$$
\begin{aligned}
\ddot{\theta} & =\frac{1}{r}\left[-2 \dot{\theta} \dot{r}+\frac{F_{t}}{M}\right] \\
\ddot{r} & =\frac{1}{I}\left[r \dot{\theta}^{2} M+F_{r}-T\right],
\end{aligned}
$$

where $r$ is the rope length from the kite to the dynamo on the ground, $\theta$ is the angle the rope makes with respect to the 
horizontal line, $I$ the moment of inertia of the dynamo's rotor and the cable drum, $T$ is the tension on the rope, $F_{r}$ and $F_{t}$ are respectively the radial and tangential forces acting on the kite due to aerodynamical forces and the weight $w=M g$.

The aerodynamical forces can be expressed in terms of the lift $L$ and the $\operatorname{drag} D$ as follows:

$F_{r}=L \sin \left(\theta-\alpha_{w}+\delta_{\alpha w}\right)+D \cos \left(\theta-\alpha_{w}+\delta_{\alpha w}\right)-w \sin \theta$
$F_{t}=L \cos \left(\theta-\alpha_{w}+\delta_{\alpha w}\right)-D \sin \left(\theta-\alpha_{w}+\delta_{\alpha w}\right)-w \cos \theta$

The angle of relative wind speed will be approximated by $\alpha_{w}+\delta_{\alpha w}$. When the kite speed is very high relative to the wind gust values, one has to take into account the speed of the kite, its orientation and the behavior of the wind speed $V$. The norms of the lift and drag obey the following equations:

$$
L=\frac{1}{2} \rho S v_{r}^{2} C_{L} \quad ; \quad D=\frac{1}{2} \rho S v_{r}^{2} C_{D},
$$

where $\rho$ is the air density, $S$ is the wing surface, $v_{r}$ is the relative wind speed, and the lift coefficient $C_{L}$ and drag coefficient $C_{D}$ are:

$$
\begin{gathered}
C_{L}=\frac{\partial C_{L}}{\partial \alpha}\left(\alpha_{w}+\alpha_{u}+\delta_{\alpha w}\right)+C_{L 0} \\
C_{D}=\frac{C_{L}^{2}}{\pi e \lambda}+C_{D 0}+k_{s p} \phi_{s p},
\end{gathered}
$$

where $C_{L 0}, C_{D 0}$ are constant coefficients, $e$ is Oswald's efficiency factor, $\lambda$ is the aspect ratio.

$U_{\alpha}$ is the control input of the pitch angle $\alpha_{u}$. The input $U_{\theta}$ controls the spoilers angle of opening $\phi_{s p}$, which modify the drag of the wing by adding a value equal to $k_{s p} \phi_{s p}$, where $k_{s p}=\frac{\partial C_{D}}{\partial U_{\theta}}$ is the drag derivative with respect to $U_{\theta}$ (see Figure 3).

The systems has three control inputs, $U_{\alpha}, U_{\theta}$ and $U_{r}$. Equations (15), (16) and (17) represent the first order dynamic response of actuators used in the experimental platform to control the torque of the dynamo/motor, the pitch angle of the wing and the angle of opening of spoilers, respectively.

$$
\begin{aligned}
\dot{T} & =\beta_{T}\left(U_{r}-T\right) \\
\dot{\alpha}_{u} & =\beta_{a}\left(U_{\alpha}-\alpha_{u}\right) \\
\dot{\phi_{s p}} & =\beta_{s p}\left(U_{\theta}-\phi_{s p}\right)
\end{aligned}
$$

Note that $U_{r}$ controls the length of the cable using the torque of the dynamo/motor. The dynamo/motor will act as a motor or as a dynamo depending on whether it is generating power or pulling the kite down to restart a cycle.

The spoilers are used to generate drag, stabilizing the kite at the desired flight angle $\theta_{D}$. Their value of the opening can vary from 0 to 160 degrees. This type of spoilers are called 'crocodile ailerons', and were used for controlling the Northrop Grumman's B-2 stealth wing. They have very little impact on the lift coefficient. The standard position is 80 degrees (Figure 2). The amount of generated drag is approximated as proportional to the angle of opening $\phi_{s p}$, wind tunnel tests have shown that the error of this approximation is lower than $15 \%$. For every combination of AOA, $\bar{V}, \dot{r}$ and $U_{\theta}$, there exists one corresponding flight angle $\theta$ value for which the kite's tangential acceleration $\ddot{\theta}$ is equal to 0 , if flight is at all possible. This particular value is named the natural flight angle $\theta_{N}$. The value of this angle is controlled by the vertical forces $F_{v}$ and the horizontal forces $F_{h}$ :

$$
\arctan \frac{F_{v}}{F_{h}}=\theta_{N}
$$

where $F_{v}=\|(\vec{L}+\vec{D}+\vec{w}) \cdot \vec{y}\|$ and $F_{h}=\|(\vec{L}+\vec{D}+$ $\vec{w}) \cdot \vec{x} \|$.

In order to reject perturbations and follow the desired angle $\theta_{N}$ when the mean wind speed $\bar{V}$ changes, one needs to be sure that there is a value of $U_{\theta}$ that can satisfy $\theta_{N}=\theta_{D}$, and that this value of $U_{\theta}$ still has enough resources to reject perturbations. It has been experimentally observed that the kite can reject perturbations using 50 percent of $U_{\theta}$. As a consequence, the values of $\theta_{D}$ have to be chosen in the intervals between the value of $\theta_{N}$ corresponding to $U_{\theta}=25 \%$ and $U_{\theta}=75 \%$. This makes that for every $\theta_{D}$, the kite has still enough margin in the control input $U_{\theta}$ to stabilize itself.

The horizontal relative wind speed (19) and the vertical airspeed (20) depend on the movements of the kite and are given with respect to $V$. In order to simplify equations, we will express the relative wind speed as the sum of the wind gust and the value of relative wind speed that would be seen by the kite with no wind gusts. In the absence of wind gust, the horizontal $v_{h}$ and the $v_{v}$ are given by:

$$
\begin{gathered}
v_{h}=\bar{V}+r \sin (\theta) \dot{\theta}-\dot{r} \cos (\theta) \\
v_{v}=r \cos (\theta) \dot{\theta}+\dot{r} \sin (\theta)
\end{gathered}
$$

Using (19) and (20) we obtain the wind angle $\alpha_{w}$ which is the angle of the wind velocity vector measured with respect to the horizon, and the kite's relative wind velocity $v_{r 0}$.

$$
\begin{gathered}
\alpha_{w}=\arctan \frac{r \cos (\theta) \dot{\theta}+\dot{r} \sin (\theta)}{\bar{V}+r \sin (\theta) \dot{\theta}-\dot{r} \cos (\theta)} \\
v_{r 0}=\sqrt{(r \cos (\theta) \dot{\theta}+\dot{r} \sin (\theta))^{2}+(\bar{V}+r \sin (\theta) \dot{\theta}-\dot{r} \cos (\theta))^{2}}
\end{gathered}
$$

Adding wind gusts perturbations, $v_{r}$ is bounded as follows:

$$
v_{r 0}-\delta_{V \max }<v_{r}<v_{r 0}+\delta_{V \max }
$$

\section{Flight Plan Design}

The flight plan has to be designed so that it respects the four safe flight conditions (Section V) and be able to follow the desired trajectory. The proposed strategy for designing the flight plan will consist in computing all the possible trajectories and choose one that satisfies the safety requirements, i.e. avoid the crashes studied previously. The possible 
trajectories will be categorized in three different domains: the 'static flight domain', when the kite's position is static, the 'constant speed flight domain' for all the straight trajectories at constant $\dot{r}$ speed, and the 'transitions flight domain', when accelerated trajectories will be studied. This particular way of studying the flight can be extended and adapted to other systems. In more complex flights, the variations of potential energy, $L / D$ ratios with respect to lateral controls, and the angle of drift, can lead to complex situations for which the possibility of flight has to be proved.

\section{A. Static flight domain}

Using the safe flight conditions, we can compute the values of AOA and relative wind speed that respect the four safety conditions when the kite's position is static (Figure 4).

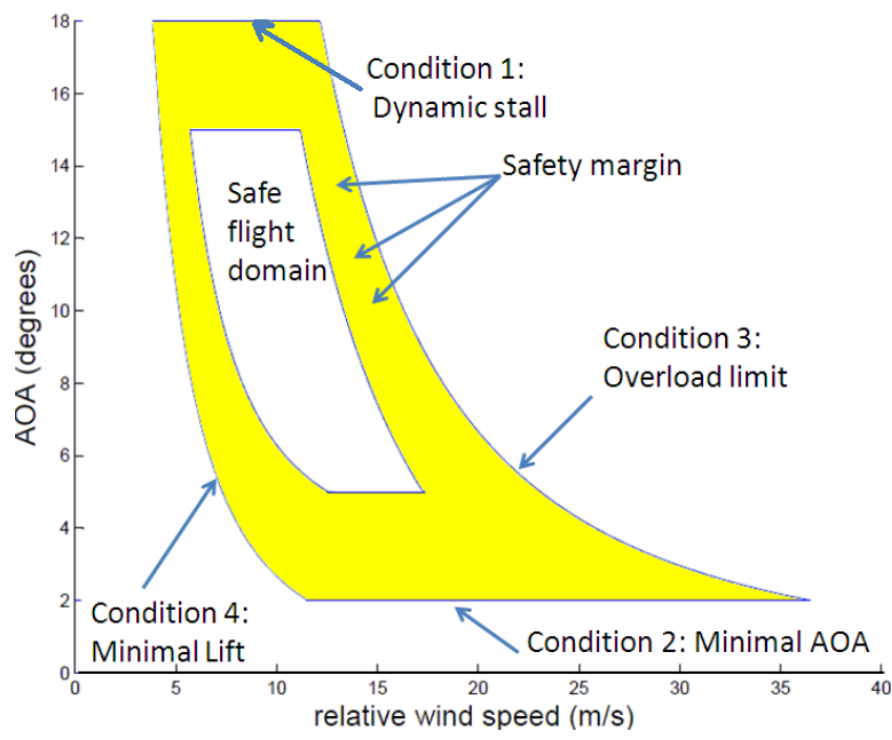

Fig. 4. Static flight domain

This domain gives a first idea of the safety-related restrictions one must consider. The values of the AOA and mean wind speed must remain in the safe flight domain. One can see that the size of the safety margins reduces the possibilities for flight and that the safe flight domain is surrounded by the four causes of crash. The size of the safety margins increases with the values of $\delta_{V \max }$ and $\delta_{\alpha w}$.

\section{B. Constant speed flight domain}

In order to set a flight plan and compute the output power, we need to find out what are the possible values for the flight angle $\theta$, depending on the $\dot{r}$ speed and on the wind speed. The basic flight trajectories are straight trajectories at constant flight angle $\theta$ and $\dot{r}$ speed. These trajectories must be followed even in case of mean wind speed change, such as when $\bar{V}$ goes from 8 to $9 \mathrm{~m} / \mathrm{s}$, and in case of AOA change. The kite has to be able to keep the same flight angle $\theta$ using less than $50 \%$ of the spoilers' total rate, as explained in section VI. In case of wind gusts, the kite must be able to regain the desired trajectory after the transition period. The kite must be able to stay at the desired flight angle $\theta_{D}$ for different values of AOA and mean wind speed $V$ using only the $U_{\theta}$ control. This condition reduces the size of the flight domain, but it is a first approach that guarantees flight safety.

Figure 5 shows the possible angles of flight corresponding to rope's speed $\dot{r}=0.5 \mathrm{~m} / \mathrm{s}$ and mean wind speeds of 8 and $9 \mathrm{~m} / \mathrm{s}$. Wind gusts are set at their less advantageous values. For each graph, the upper curve corresponds to the $\theta_{N}$ value corresponding to $U_{\theta}=25 \%$ and the lower to the $\theta_{N}$ value corresponding to $U_{\theta}=75 \%$.
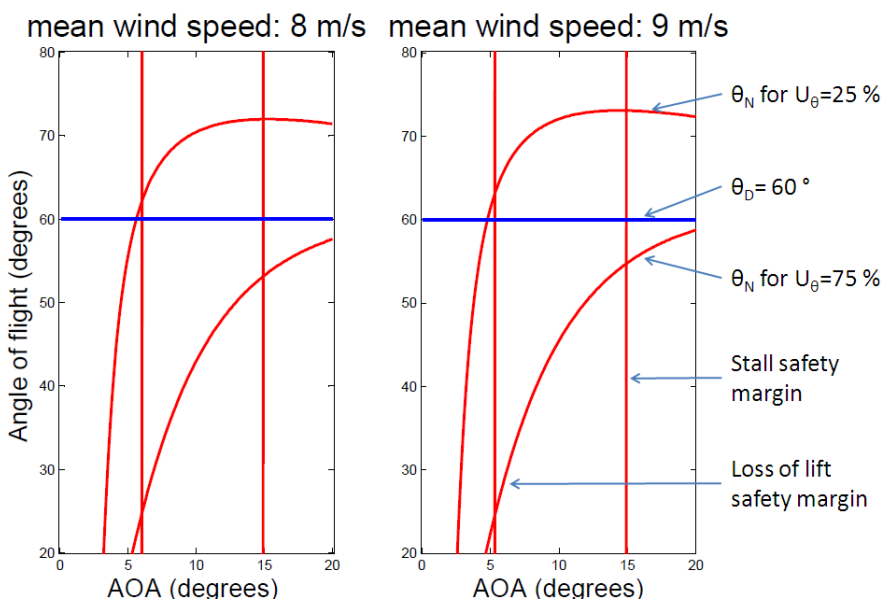

Fig. 5. Possible flight angle $\theta$ values depending on the AOA value, for $\dot{r}=0.5 \mathrm{~m} / \mathrm{s}$.

We can see that with $\dot{r}=0.5$, the $\theta_{D}$ value can take any value between 54 and 63 degrees. The values of $\theta_{D}$ have to be chosen so that they are compatible with the upward and downward speeds. This condition is verified for the values given in Table I.

TABLE I

COEFFICIENTS OF THE EXPERIMENTAL SET-UP

\begin{tabular}{lll}
\hline Variable & production phase value & recovery phase value \\
$\dot{r}$ & $0.1 \mathrm{~m} / \mathrm{s}$ & $0.2 \mathrm{~m} / \mathrm{s}$ \\
$\alpha$ & 15 degrees & 6 degrees \\
$r$ & $1 \mathrm{~m}$ (max value) & $0.2 \mathrm{~m}$ (min value) \\
$\theta_{D}$ & 65 degrees & 65 degrees \\
\hline
\end{tabular}

Vertical lines in Figure 5 are the limits on AOA to respect Conditions 1 (avoid stall) and 2 (avoid frontal collapse). Taking into account the safety margins, the angle of attack has to be greater than 5.2 degrees for $8 \mathrm{~m} / \mathrm{s}$ of mean wind speed, and 6 degrees for $9 \mathrm{~m} / \mathrm{s}$. For these ranges of wind speeds and AOA, Conditions 3 (overload) and 4 (minimal lift) are respected as well.

\section{Accelerated flight domain}

This study will focus on constant flight angle trajectories. In order to keep a tense tether, one needs to satisfy the two following conditions. First, in the case where the acceleration $\ddot{r}$ is positive, the maximal acceleration of the rope must be lower than the kite's acceleration, else the cable will go loose. 
The kite acceleration rate can be approximated by $\|\vec{L}\|$ $/ M$. This condition can be written as follows:

$$
\ddot{r}<\frac{\|\vec{L}\|}{M}
$$

\section{MAXIMUM ENERGy PRODUCTION}

The maximum energy production is studied using the following six parameters: the upwards speed $\dot{r}_{1}$ and the radial force $F_{r 1}$ of the kite during the production phase, the downwards speed $\dot{r}_{2}$ and the radial force $F_{r 2}$ of the kite during the recovery phase, and the minimum and maximum rope length ( $r_{\min }$ and $r_{\max }$, respectively) attained at the time $t_{1}$ and $t_{2}$.

The amount of energy produced during a cycle of this flight plan is given by:

$$
E\left(t_{0}, t_{1}, t_{2}\right)=\int_{t_{0}}^{t_{1}} F_{r 1} \dot{r}_{1} d t+\int_{t_{1}}^{t_{2}} F_{r_{2}} \dot{r}_{2} d t
$$

where the first term is the energy produced during the production phase, and the second is the energy consumed while reeling the kite back in.

The mean power can be written as:

$$
P\left(t_{0}, t_{1}, t_{2}\right)=\frac{E\left(t_{0}, t_{1}, t_{2}\right)}{t_{2}-t_{0}}
$$

Note that this simple flight plan and the energy expression do not take into account the loss of energy due to the transition phases, the efficiency of the motors and actuators, the rope's weight or friction losses. This kite could have a maximum production of $50 \mathrm{~W} / \mathrm{m}^{2}$ with a mean wind speed of $8 \mathrm{~m} / \mathrm{s}$, an upward speed $\dot{r}_{1}$ of $5 \mathrm{~m} / \mathrm{s}$ and a reeling speed of of $15 \mathrm{~m} / \mathrm{s}$. The experimental setup has a maximum rope length $r$ of one meter, and as a consequence, it cannot reach high values of $\dot{r}$. The maximal values that can be reached are about $0.3 \mathrm{~m} / \mathrm{s}$. The performance of recovery phases with these values of $\dot{r}$ are quite low, and one needs to keep a minimal angle of attack of approximately 6 degrees. As a result, the following flight plan has been designed (Table I). It respects all the safety conditions and leads to a maximal output power of approximately $0.3 \mathrm{~W}$.

\section{Kite Control Algorithms}

During last decade, kite systems have been studied by many different automatic control teams. Nonlinear model predictive control (NMPC) has been applied in [17], [15], [14]. Trajectory tracking methods including neural network control [16], robust control [18], direct-inverse control [19], and nonlinear adaptive tracking control [11] has been also proposed. For more details on the different control approaches the reader can refer to the recent survey [13]. This study highlights the fact that respecting some conditions on flight mechanics and using simple flight plans one can make use of simple control algorithms.

\section{A. Proposed control strategy}

In the near future, kites system will probably be used in wind power farms with great constraints on the proximity of neighboring kite power systems. Management of the position of the kite and the timing of the energy production phases will also be of great importance. That is why the choice has been made to design a control strategy that allows us to easily control the kite's position and the duration of each phase. This section will propose a control strategy that, once the $\dot{r}$ and the $\theta$ values are properly controlled, can control the output power using as only input the angle of attack control. This enables the use of very simple control algorithms. The general control scheme is shown in Figure (6).

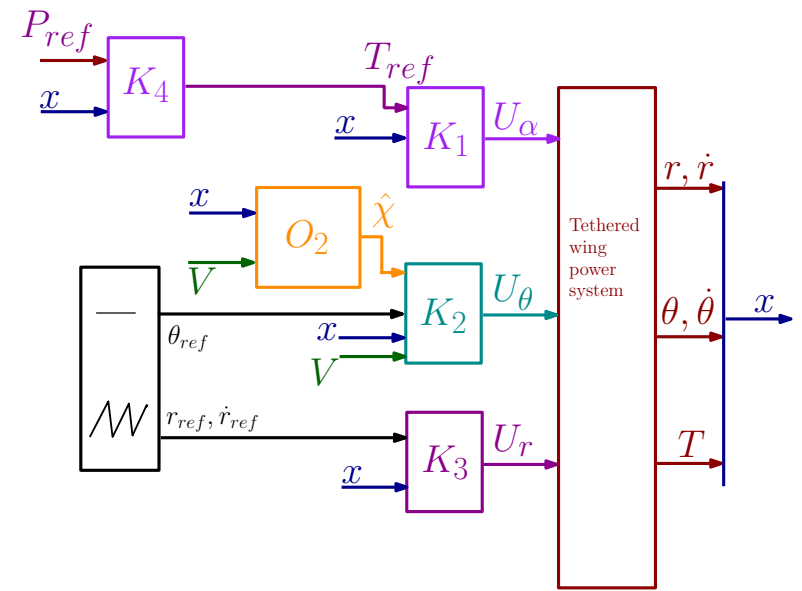

Fig. 6. The complete system: $\mathrm{O}_{2}$ is the observer of the expression $\chi$ defined by (30). $K_{1}, K_{2}, K_{3}$ and $K_{4}$ are respectively feedback controlers for $\alpha, \theta, r$ and $\mathrm{P}$.

\section{Control of flight angle $\theta$}

The control of the flight angle can be done with simple control algorithms if the measurement of $\theta$ is accurate enough. The Gipsa-lab control system uses a sensor that is not very accurate, and which gives a numerical signal not easily used for the stabilization of the flight angle $\theta$. The proposed solution reconstitutes $\theta$ and $\dot{\theta}$ using the following observer-based strategy. Note that the equation (8) can be rewritten in the following condensed form:

$$
\ddot{\theta}=\chi+Q U_{\theta},
$$

where $Q$ and $\chi$ are given by the following expressions:

$$
\begin{aligned}
Q= & -\frac{\rho S v_{r}^{2} k_{s p}}{2 M r} \sin \left(\theta-\alpha_{w}\right) \\
\chi= & -\frac{2 \dot{r} \dot{\theta}}{r}+\frac{L \cos \left(\theta-\alpha_{w}\right)-P \cos \theta}{r M} \\
& -\frac{\rho S v_{r}^{2}\left(\frac{C_{L}^{2}}{\pi e \lambda}+C_{D_{0}}\right) \sin \left(\theta-\alpha_{w}\right)}{2 r M}
\end{aligned}
$$

Note that $\chi$ is a badly known term. That is the reason why $\chi$ is hereafter estimated using the following Luenberger estimator, based on the sole measurement of $\theta$ : 


$$
\begin{aligned}
\hat{\chi} & =\left(\begin{array}{lll}
0 & 0 & 1
\end{array}\right) \hat{X}_{1} \\
\dot{\hat{X}}_{1} & =\left(A_{1}-L_{1} C_{1}\right) \hat{X}_{1}+B_{1} U_{\theta}+L_{1} \theta,
\end{aligned}
$$

where the observer's state is $X_{1}=[\theta \dot{\theta} \chi]$ and

$$
A_{1}:=\left(\begin{array}{lll}
0 & 1 & 0 \\
0 & 0 & 1 \\
0 & 0 & 0
\end{array}\right) ; B_{1}:=\left(\begin{array}{l}
0 \\
Q \\
0
\end{array}\right) ; C_{1}:=\left(\begin{array}{ll}
1 & 0
\end{array}\right.
$$

The matrix gain $L_{1}$ is the observer gain obtained using LQE design. This gives the control law given by (31)-(32) together with

$$
u_{\theta}=-\frac{1}{Q}\left[\hat{\chi}+\lambda_{1_{\theta}}\left(\dot{\theta}-\dot{\theta}_{r e f}\right)+\lambda_{2_{\theta}}\left(\theta-\theta_{r e f}\right)\right],
$$

where $\theta_{\text {ref }}$ and $\dot{\theta}_{\text {ref }}$ are respectively the desired angle and angular velocity while $\lambda_{1_{\theta}}$ and $\lambda_{2 \theta}$ are design parameters. Note that the feedback law (34) only needs the estimation of $\theta, \dot{\theta}$ and $\chi$ and does not require any particular knowledge on the drag force expression.

Control of the rope's length $r$ The rope's length $r$ control works like a servo motor using a torque control as input. the $K_{3}$ PID controller has been implemented for this task. It must be adapted so that it can respect the two following conditions. First, it must respect (24) on the maximal value for the acceleration $\ddot{r}$. Second, the rope length $r$ and the kite's traction should be decoupled enough so that the $r$ control can work without being affected by the traction of the kite.

\section{Control of the mean output power}

The proposed output power control uses the controller $K_{4}$ that computes the desired tension, which is controlled by the $K_{1}$ controller using $U_{\alpha}$. This kite system can not produce a constant desired output power $P_{\text {ref }}$ because it consumes power during the recovery phase. The aim of this algorithm will be to control the produced amount of energy of each cycle. The cycle duration is $t_{c y c l e}$, the produced energy is $E_{\text {cycle }}$. For simplicity, $P_{\text {ref }}$ will be supposed constant, but this algorithm can be adapted to varying values of $P_{r e f}$. The proposed algorithm will control the desired energy $E_{\text {real }}$ in order to reach the desired energy $E_{\text {des }}$ defined by the following definition:

$$
\begin{array}{r}
E_{\text {des }}=P_{\text {ref }} t_{\text {cycle }} \\
E_{\text {real }}=E_{\text {rec }}+E_{\text {prod }},
\end{array}
$$

where $E_{\text {prod }}$ is the energy produced during the production cycle and $E_{r e c}$ the energy consumed during the recovery phase. The proposed algorithm is based on the following simple rules:

1) As the kite performs its recovery phase, the consumed power is measured.

2) At the end of the recovery cycle, the $E_{\text {prod }}$ value needed to satisfy $E_{\text {real }}=E_{\text {des }}$ is defined by: $E_{\text {prod }}=E_{\text {des }}-E_{\text {rec }}$ 3) To produce the desired amount of energy at the end of the production phase, the traction force has to be $T_{\text {des }}=\left(E_{\text {des }}-E_{\text {rec }}\right) / \dot{r}$

The traction force is measured by a torque sensor. It is equal to the torque divided by the radius $R$ of the drum. The traction force can be approximated as proportional to $\alpha v_{r}^{2}$. The $v_{r}$ values are steady enough to use a simple PID controller. Nevertheless, it must be bounded so that it respects the four safe flight conditions [chapter V] .

\section{Experimental Setup and Validation}

The experimental setup ${ }^{1}$ has been described in [20]. Briefly, it is composed of a wind tunnel that generates a controlled windflow that goes up to $9 \mathrm{~m} / \mathrm{s}$, the dynamo/motor and its cable drum. Controllers are implemented on the experimental set-up using the XPC target real-time toolbox of Matlab. The coefficients of the experimental set-up can are given in Table II.

TABLE II

COEFFICIENTS OF THE EXPERIMENTAL SET-UP

\begin{tabular}{lll}
\hline Symbol & Name & Value \\
$M$ & mass & $0.08 \mathrm{Kg}$ \\
$I$ & moment of inertia & $0.0481 \mathrm{Kg} \mathrm{m}$ \\
$\rho$ & air density & $1.225 \mathrm{Kg} / \mathrm{m}^{3}$ \\
$S$ & wing area & $0.14 \mathrm{~m}^{2}$ \\
$e$ & Oswald's factor & 0.7 \\
$\lambda$ & aspect ratio & 2.5 \\
$\frac{\partial C L}{\partial \alpha}$ & lift derivative w.r.t. $\alpha$ & $0.07 \mathrm{deg}^{-1}$ \\
$C_{D 0}$ & zero lift drag & 0.01 \\
$C_{L 0}$ & lift coefficient at $\alpha=0$ & 0 \\
$V$ & mean air speed & $8-9 \mathrm{~m} / \mathrm{s}$ \\
$k_{s p}$ & drag derivative w.r.t. $\phi_{s p}$ & $0.003 \mathrm{deg}$ \\
$\beta_{s p}$ & inverse of the time constant & $100 \mathrm{~s}^{-1}$ \\
$\beta_{a}$ & inverse of the time constant & $100 \mathrm{~s}^{-1}$ \\
$\beta_{T}$ & inverse of time constant & $14.28 \mathrm{~s}^{-1}$ \\
\hline
\end{tabular}

Figure 7 shows the efficiency of the control of the flight angle in rejecting strong manual disturbances introduced by the operator. During this scenario, the experimenter pushes the kite very roughly. This kind of perturbations might seem over sized, but one has to keep in mind that wind gusts and tether vibrations can provoke significant disturbances to the kite. These are the tests that kites systems must pass in order to be considered as a safe system.

Figure 8 shows the ability of the proposed control scheme to achieve mean power tracking under unmeasured and varying wind speed from 7.5 to $9 \mathrm{~m} / \mathrm{s}$. One can see the corresponding actuator's variations. The average level of accuracy for the power tracking is higher than 95 percent, even with strong wind gusts. As the cycle's length is constant and the produced output power is well controlled, several

\footnotetext{
${ }^{1} \mathrm{~A}$ movie showing the experimental results can be viewed at http://www.gipsa-lab.grenoble-inp.fr/recherche/platesformes.php?id_plateforme $=70$
} 


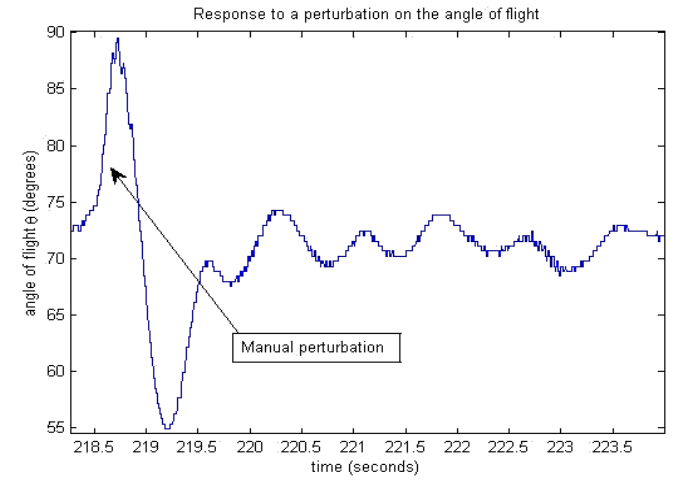

Fig. 7. Manual perturbation. Note that the kite is stabilized within 2 seconds.
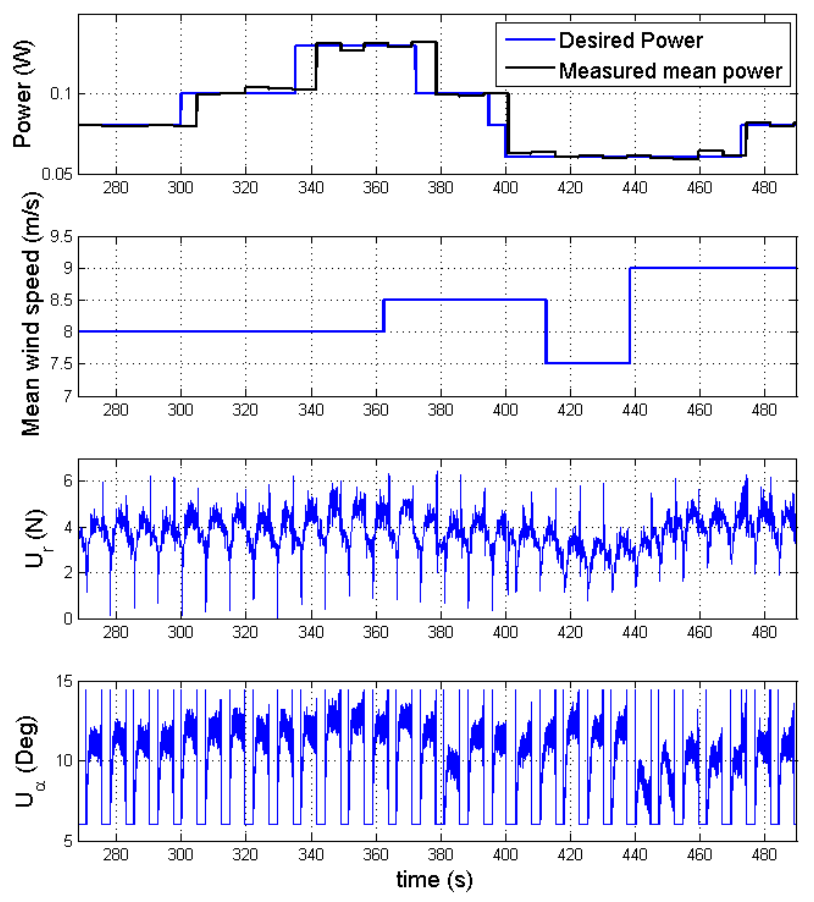

Fig. 8. Experimental results showing the mean power tracking capability of the proposed control framework under unmeasured and varying wind speed.

coordinated kites systems could produce a constant amount of energy.

\section{Conclusion}

In this paper, a part of the ongoing work at Gipsa-lab Grenoble on kite systems is presented following a rational experimentally-based observer-oriented control design. The indoor testing made the tests faster, safer, and with more accurate results. The simulations and the experimental results fit quite well, which validates the aerodynamical model and the effectiveness of the proposed control algorithms. Our ongoing work is focused on the full $3 \mathrm{D}$ control of a kite

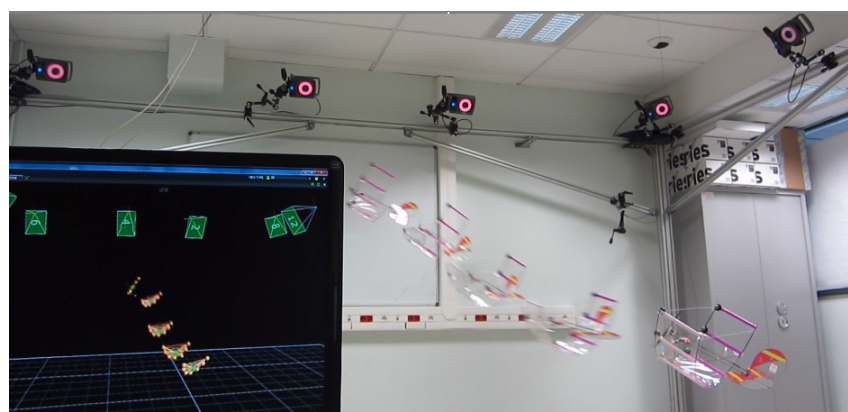

Fig. 9. 3D flight prototype using the Vicon@Cmotion capture system.

system, see figure 9. The experimental setup is similar to the one presented in this paper, but it has an extra degree of freedom for lateral control. The control algorithms use the measurements given by a Vicon (c) system. The other experiment our team is working on consists of performing 'reverse pumping'. This principle allows the kite to stay in flight even when there is no wind.

\section{REFERENCES}

[1] Wellington, A. (1893), Mechanics of flight and aspiration, Proceedings of the International conference on Aerial Navigation, Chicago.

[2] Gastine, L. (2010), L' A. B. C. de l'aviation: Biplans et monoplans.

[3] Lilienthal, O. (1911), The bird flight as the basis of aviation.

[4] Crouch, T. \& Jakab P. (2003), The Wright brothers and the invention of the aerial age, Nationnal geographic society.

[5] Almond, P. (1997), Aviation the early years, Getty images.

[6] Ellot, B. (2000), Bleriot: Herald of an age, Arcadia Publishing.

[7] Don Dwiggins (1979), Man-powered aircraft, Tab Books.

[8] Grosser, M. (2004), Gossamer odysey, The thiumph of Human powered flight, Zenith press.

[9] Conner, M. (2001), Hans Von Ohain: Elegance in flight, American Institute of Aeronautics and Astronautics.

[10] Anderson, J. (2001), Fundamental of aerodynamics, McGraw-Hill.

[11] Baayen, J. (2011), Automatic trajectory tracking control of kites, $\mathrm{PhD}$ of Delft University of Technology.

[12] French Federation of Paragliding Website, http://federation.ffvl.fr/

[13] Ahmed, M. and Hably, A. and Bacha, S.(2012), High Altitude Wind Power Systems: A Survey on Power Kites, The International conference on electrical machines.

[14] Ahmed, M. and Hably, A. and Bacha, S. (2011), Power Maximization of a Closed-orbit Kite Generator System, 50th Conference on Decision and Control and European Control Conference (IEEE CDC-ECC).

[15] Canale, M. et al. (2010), High Altitude Wind Energy Generation Using Controlled Power Kites, IEEE Transactions On Control Systems Technology, volume 18(2), pages 279-293.

[16] Furey, A. and Harvey, I. (2007), Evolution of neural networks for active control of tethered airfoils, Advances in Artificial Life Journal, pages 746-755, Springer.

[17] Ilzhofer, A. and Houska, B. and Diehl, M. (2007), Nonlinear MPC of kites under varying wind conditions for a new class of large-scale wind power generators, International Journal Of Robust And Nonlinear Control,volume 17, pages 1590-1599.

[18] , Podgaets, A. and Ockels, W. (2007), Robust Control of Laddermill Wind Energy System, Proceedings of the Wind Power Shanghai Conference 2007.

[19] Novara, C. and Fagiano, L. and Milanese, M. (2011), Direct datadriven inverse control of a power kite for high altitude wind energy conversion, IEEE International Conference on Control Applications (CCA), pages 240-245.

[20] Lozano, R. Jr and Alamir, M. and Dumon, J. and Hably, A. (2011),Control of a wind power system based on a tethered wing, IFAC EGNCA, Bangalore, India 2011

[21] Timmer, W.A. (2008), Two-dimensional low-Reynolds number wind tunnel results for airfoil NACA 0018 , pages 525-537, Wind Engineering, Volume 32, Number 6 / December 2008 